\title{
Application of Lorentz force eddy current testing and eddy current testing on moving nonmagnetic conductors
}

\author{
Matthias Carlstedt*, Konstantin Porzig, Robert P. Uhlig, Mladen Zec, Marek Ziolkowski and \\ Hartmut Brauer \\ Department of Advanced Electromagnetics, Technische Universität Ilmenau, Helmholtzplatz, Ilmenau, \\ Germany
}

\begin{abstract}
Lorentz force eddy current testing is a novel nondestructive testing technique which can be applied preferably to the identification of internal defects in non-ferromagnetic moving conductors. This paper describes the comparison of this new technique with well-known eddy current testing. Measurements and numerical simulations have been done for both techniques for artificial subsurface defects in a test specimen made of Aluminum alloy moving with constant velocity.
\end{abstract}

Keywords: Eddy current testing, lorentz force eddy current testing, moving conductors, nondestructive testing

\section{Introduction}

Nondestructive Testing (NDT) and Evaluation (NDE) of electrically conductive objects require reliable methods to detect material anomalies or defects. Among the electromagnetic inspection techniques classical eddy current testing (ECT) has found a wide range of application in industry [1,5], also due to the low demands to the measurement environment. However, the detection of subsurface defects is mainly limited by the frequency dependent penetration depth of eddy currents. Furthermore, the applicable testing speed is strongly restricted depending on the properties of defects, e.g. dimensions and conductivity.

With Lorentz force eddy current testing (LET) a novel nondestructive testing technique has been presented [2,7], which is considered as a complementary principle of electromagnetic testing.

\section{Basic principle of LET and ECT}

LET is a nondestructive testing technique to detect material anomalies and defects in conductive nonferromagnetic materials. It is based on generating eddy currents by setting a permanent magnet (PM),

\footnotetext{
${ }^{*}$ Corresponding author: Matthias Carlstedt, Department of Advanced Electromagnetics, Technische Universität Ilmenau, Helmholtzplatz 2, D-98693, Ilmenau, Germany. E-mail: matthias.carlstedt@tu-ilmenau.de. 


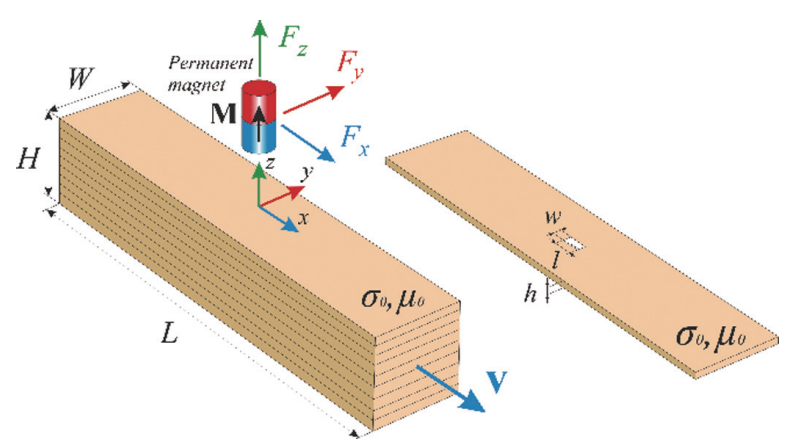

Fig. 1. Solid conductive bar as test specimen, approximated by a set of thin sheets moving with constant velocity below a permanent magnet (left) and representation of an artificial defect (slit) in one single sheet for variation of defect (right).

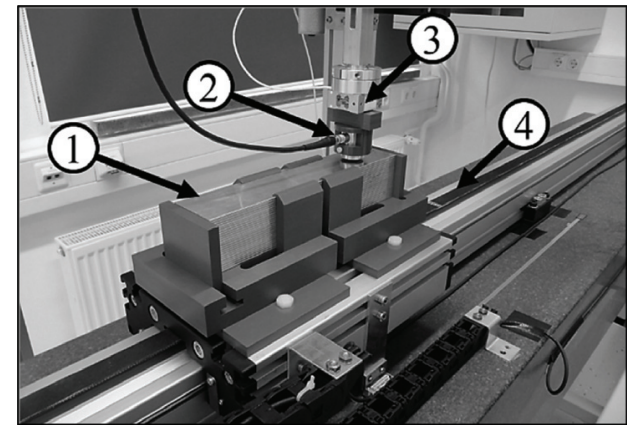

Fig. 2. Experimental setup: detail view on moving specimen (1), ECT probe (2), force sensor (3) and linear drive (4).

providing a stationary primary magnetic field $\mathbf{B}$, into relative motion to the object under test (see Fig. 1). Due to the relative movement, eddy currents are induced governed by Ohm's law for moving conductors

$$
\mathbf{J}=\sigma\left(-\frac{\partial \mathbf{A}}{\partial t}-\nabla \varphi+\mathbf{v} \times \mathbf{B}\right)
$$

which are accompanied with a Lorentz force $\mathbf{F}$, acting on the specimen. By Newton's third axiom, an equivalent force

$$
\mathbf{F}=\int_{V} \mathbf{J} \times \mathbf{B} d V
$$

is acting on the permanent magnet. In contrast, classical eddy current testing techniques use an alternating current driven coil to generate a time changing magnetic field which induces eddy currents while interacting with an electrically conducting specimen.

The governing equation for both, ECT and LET is the magnetic field transport equation [3]. In potential form, it is given by

$$
\nabla \times\left(\frac{1}{\mu_{0}} \nabla \times \mathbf{A}-\mathbf{M}\right)=-\sigma\left(\frac{\partial \mathbf{A}}{\partial t}+\nabla \varphi-\mathbf{v} \times \nabla \times \mathbf{A}\right)+\mathbf{j}_{e}
$$

where A denotes the magnetic vector potential, $\varphi$ the scalar electric potential, $\mathbf{M}$ the magnetization of the permanent magnet, $\mathbf{j}_{e}$ the external current density, and $\mathbf{v}$ the velocity of the specimen.

In case of ECT, the diffusion term $\partial \mathbf{A} / \partial t$ dominates. However, in LET eddy currents are induced by the convection term $\mathbf{v} \times(\nabla \times \mathbf{A})$. In the vicinity of a defect, the magnetic field in both techniques is perturbed in time, resulting in an additional $\partial \mathbf{A} / \partial t$ term.

For both techniques the detection of subsurface defects is mainly limited by the penetration depth of eddy currents in the specimen. In ECT the frequency dependent skin-depth

$$
\delta=\sqrt{\frac{2}{\omega \mu \sigma}}
$$

results in a fast decay of the desired signal limiting the detection of subsurface defects. By transforming the magnetic convection diffusion equation in its' non-dimensional form [3], the magnetic Reynolds number $R_{m}$ can be derived as

$$
R_{m}=\mu \sigma v L
$$


where $L$ is the typical length-scale of the problem. In case of $R_{m} \ll 1$, the resulting field is primarily determined by the primary magnetic field. In case of $R_{m} \gg 1$, the magnetic field is gradually expelled from the conductor which results in a similar phenomenon as skin effect in Eq. (4).

\section{Methods}

\subsection{Methodology for comparison}

For the comparison of both techniques a systematic procedure had to be pursued, using numerical simulations and experiments. Due to the similarity of the underlying physical effects, the use of adequate sensors and settings and the formulation of appropriate criteria for comparison are required. Because LET is aiming to diminish the limitations in detecting subsurface defects, the preselection of appropriate ECT probes is the first important step for the comparison. For that purpose, a specimen of $250 \times 50$ $\times 50 \mathrm{~mm}^{3}$ out of Aluminum with well-known artificial defects in different depths has been used. Due to the limitations of the used ECT device (Elotest N300, Rohmann $\mathrm{GmbH}$ ), only one of the available probes met the desired performance to detect defects in depths deeper than $5 \mathrm{~mm}$ with wanted lateral resolution.

The second important step for the comparison was the reverse-engineering of the chosen ECT probe (PKA48, Rohmann $\mathrm{GmbH}$ ) using high-resolution X-ray images, measurements of the magnetic flux density and numerical simulations to estimate material properties. Finally, both methods were compared in experimental und numerical studies on a chosen testing problem with artificial defects.

\subsection{Experimental setup}

The experimental setup used to measure the Lorentz force acting on the magnet consists of four main components, a belt driven linear drive, a permanent magnet, a force sensor and a positioning stage. The linear drive realizes the relative motion between magnet and specimen with a repetition accuracy lower than $\pm 0.2 \mathrm{~mm}$ at a maximum velocity of $3.75 \mathrm{~m} / \mathrm{s}$ with an acceleration of up to $20 \mathrm{~m} / \mathrm{s}^{2}$. The permanent magnet provides the stationary primary magnetic field. The chosen permanent magnet for comparison is a cylindrical $\mathrm{NdFeB}$ magnet with a material grade of $\mathrm{N} 38$, a diameter of $15 \mathrm{~mm}$ and a height of $25 \mathrm{~mm}$. The used force sensor is a 3D force sensor based on strain gauge technology with a resolution in moving direction $(x)$ and side direction $(y)$ of $15 \mathrm{mN}$ and $50 \mathrm{mN}$ in lift-off direction $(z)$.

For the alignment of the permanent magnet in $y$ - $z$-plane, a 2D positioning stage is used which is driven by two stepper drives providing a positioning accuracy of $\pm 0.05 \mathrm{~mm}$ over a range of $45 \mathrm{~mm}$ each.

The entire communication with all control units and the data acquisition of the force sensor are done using a PXI system by National Instruments. For ECT measurements, the permanent magnet has been replaced by a fixture for ECT probes and a commercial ECT device has been added to the experimental setup. The tested specimen chosen for comparative investigation of artificial defects, is a stack of 25 Aluminum sheets each $250 \times 50 \times 2 \mathrm{~mm}^{3}$.

\subsection{Numerical model}

A numerical model was constructed to compare measurements with simulations of the given setups. For both techniques, the same numerical strategy was used, namely, the finite element method (FEM) with the moving defect approach (MDA) as described in [8] and illustrated in Fig. 3 for ECT. The MDA 
introduces a moving defect region where the defect position is altered in time by defining the material properties by means of logical expressions, whereas the whole geometry is at rest. The advantage of this approach is that no remeshing or interpolation is needed between successive time-steps which leads to a significant reduction of computation time. This method is only applicable if the defect is far away from the outer boundary.

In order to avoid numerical inaccuracies, in case of LET, the moving defect region has to be meshed according to the relative velocity between PM and the conductor causing that the time-step $\Delta t$ used in the transient solver has to be chosen depending on the mesh size $\Delta x$ in the motion direction. In case of LET the mesh size was defined to be $\Delta x=1 \mathrm{~mm}$, resulting in a time-step of $\Delta t=2 \mathrm{~ms}$ for a velocity of $0.5 \mathrm{~m} / \mathrm{s}$. In contrary, in case of ECT (time-domain implementation), the time-step $\Delta t$ has to be defined first assuring proper modelling of the signals. It is defined by the applied frequency $f$ such as each period is sampled by 40 points $\Delta t=1 /(40 f)$ to ensure numerically stable simulations. That implies a mesh size of $\Delta x=125 \mu \mathrm{m}$ and a time-step of $\Delta t=0.25 \mathrm{~ms}$ for a frequency of $100 \mathrm{~Hz}$ and a velocity of $0.5 \mathrm{~m} / \mathrm{s}$.

In general a time dependent approach for ECT should be applied if the ratio between velocity and frequency $v / f$ is in the range of defect size. This ratio states the moved distance of the ECT-coil during one sinusoidal period. Otherwise, enough periods are present during the time the defect passes the ECTcoil localization and a quasi-static approach in the frequency domain can be used.

All LET simulations were performed in 3D. However, ECT simulations were done in 2D due to the increased number of finite elements to model the ECT-coil and the smaller time-step size.

The ECT problem was solved using a magnetic vector potential formulation in the whole domain due to the two-dimensionality of the problem. In contrast, the 3D LET problem was solved using a scalar magnetic potential $\Psi$ formulation outside the conductor, and a modified magnetic vector potential $\mathbf{A}^{*}$ formulation inside the conductor, whereas $\mathbf{A}^{*}$ contains the electric scalar potential $\varphi$ [9]

$$
\mathbf{A}^{*}=\mathbf{A}+\int_{0}^{t} \nabla \varphi d t .
$$

As a result, the scalar electric potential $\varphi$ is excluded from Eq. (3) and the number of degrees of freedom per node is reduced from 4 to 3 inside the conducting region. In addition, the symmetry of the problem allows a reduction to one half of the total geometry by applying the boundary conditions $\mathbf{n} \times \mathbf{A}^{*}=\mathbf{0}$ inside the conductor and $\mathbf{n} \cdot \mathbf{B}=0$ outside the conductor.

\section{Results and discussion}

In the following, a comparison between numerical and measured results for both, ECT and LET for various testing velocities and defect depths is presented. In both settings, the lift-off distance between the permanent magnet or ECT probe and specimen was kept constant at $1 \mathrm{~mm}$.

The measured signals of the ECT device were recorded, low pass filtered, drift corrected, synchronized, $y$-shifted, and normalized to the maximum defect perturbation amplitude calculated numerically due to the lack of any quantitative information.

The digital signal processing of the raw signals of LET measurements consist of a wavelet filter, drift correction, synchronization and scaling. Additionally, the force components $F_{c}$ with $c \in\{x, y\}$ were subtracted by their steady components $F_{c 0}$ and normalized to the constant drag force without defect $F_{0}$ at $0.7 \mathrm{~m} / \mathrm{s}$.

$$
\Delta F_{c}=\frac{F_{c}-F_{c 0}}{F_{0}} 100 \%
$$


Table 1

Parameters of the experimental setup and numerical model

\begin{tabular}{lll}
\hline Par. & Value & Description \\
\hline$f$ & $100[\mathrm{~Hz}]$ & Excitation frequency (ECT) \\
$v$ & $0.3-0.7[\mathrm{~m} / \mathrm{s}]$ & Velocity of specimen \\
$B_{r}$ & $1.17[\mathrm{~T}]$ & Remanence of permanent magnet \\
$\sigma_{\text {bar }}$ & $30.61[\mathrm{MS} / \mathrm{m}]$ & Electrical conductivity of specimen \\
1 & $1[\mathrm{~mm}]$ & Lift-off distance \\
$R_{\text {mag }}$ & $15[\mathrm{~mm}]$ & Diameter of permanent magnet (LET) \\
$H_{\text {mag }}$ & $25[\mathrm{~mm}]$ & Height of permanent magnet (LET) \\
$L$ & $25[\mathrm{~mm}]$ & Typical length scale (LET) \\
$R_{p}$ & $8.9[\mathrm{~mm}]$ & Outer radius of primary windings (ECT) \\
$R_{s}$ & $7.1[\mathrm{~mm}]$ & Outer radius of secondary windings (ECT) \\
$\sigma_{\text {case }}$ & $37.74[\mathrm{MS} / \mathrm{m}]$ & Electrical conductivity of case (ECT) \\
$\mu_{\text {shield }}$ & 232 & Relative permeability of shield (ECT) \\
$\mu_{\text {core }}$ & 5 & Relative permeability of core (ECT) \\
\hline
\end{tabular}

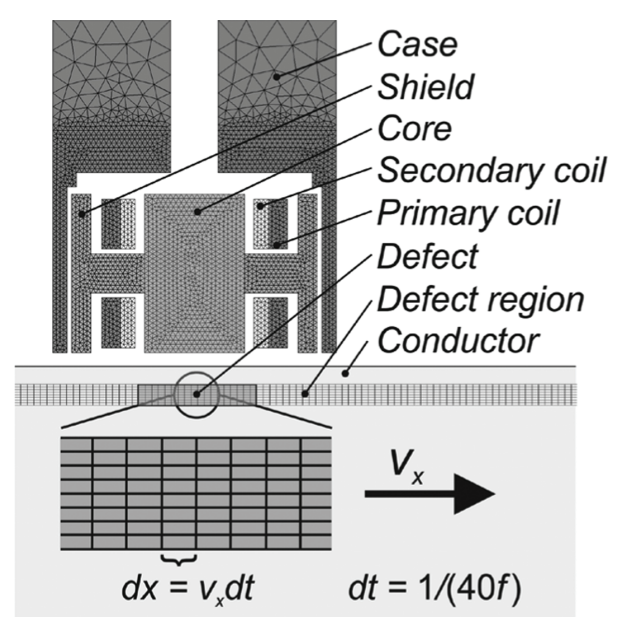

Fig. 3. Numerical FEM-model (2D) of ECT time dependent simulations.

\subsection{Velocity study}

In case of ECT, the normalized time development and the magnitude of the induced voltage in the secondary coil $U_{\text {sec }}(t)$ were analyzed at different velocities. In order to derive the instantaneous magnitude of the numerically evaluated output signal, the Hilbert-transformation of $U_{s e c}(t)$ and its magnitude were calculated.

Figure 4 illustrates how a defect affects the instantaneous magnitude and the phase of the output signal for different velocities. At low testing velocities such as $0.25 \mathrm{~m} / \mathrm{s}$ and $100 \mathrm{~Hz}$ (frequency of ECT primary signal), a sufficient amount of periods occur while the defect passes the ECT probe. In this case, the " $W$ "-shape of the output signal was obvious. The calculated normalized root-mean-square deviation Eq. (8) of $13.2 \%$ indicates an adequate agreement between the experimental und numerical results. The major cause for the observed deviation lies in the uncertainties of the velocity and sampling frequency which was provided by the ECT device. 

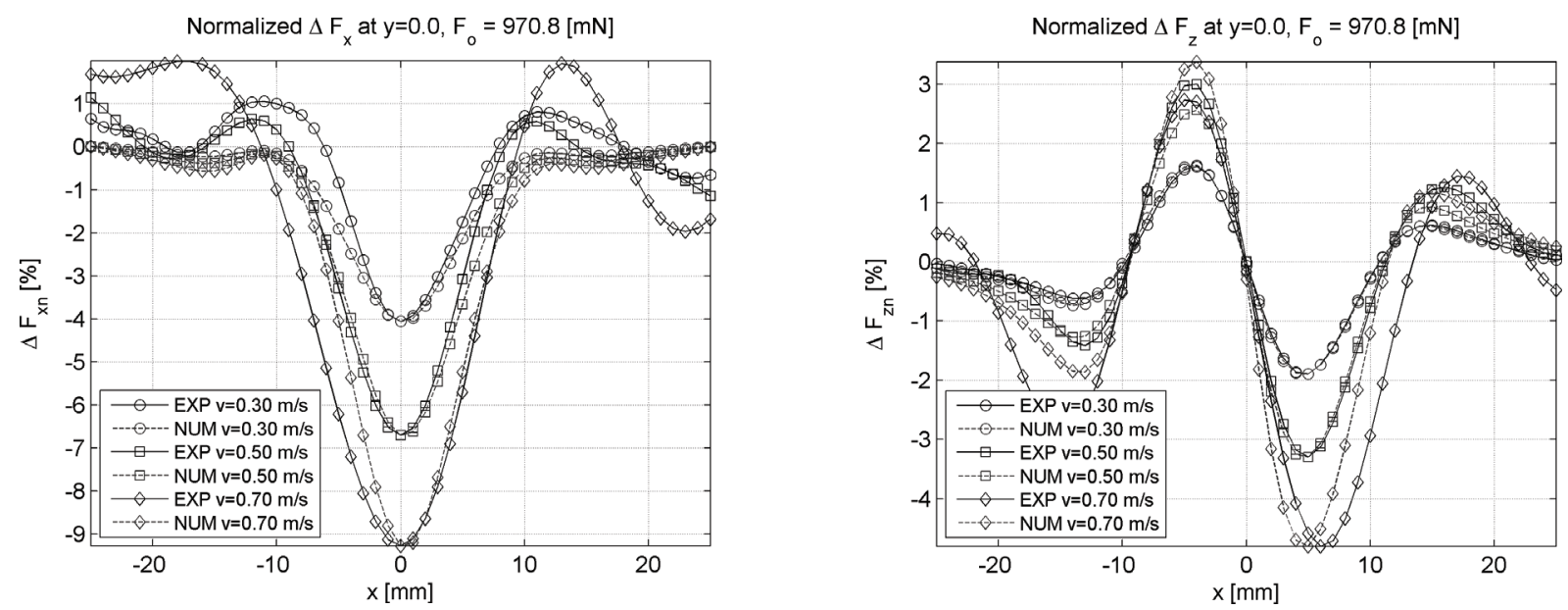

Fig. 5. Normalized forces exerting on the permanent magnet: drag force $\Delta F_{x n}$ (left), lift force $\Delta F_{z n}$ (right), measured in the vicinity of an artificial defect of size $12 \times 2 \times 2 \mathrm{~mm}^{3}$ for different testing velocities at a defect depth of $3 \mathrm{~mm}$.

In case of higher testing velocities, such as $0.5 \mathrm{~m} / \mathrm{s}$, the amount of periods of the ECT signal, interacting with the moving defect, decreases. Nevertheless, the Hilbert-transform of the numerical signal results in a similar shape as for lower speeds. However, it is noted that the measured signal shows a smoothed waveform due to a device-specific low pass filter with cutoff frequency of $10 \mathrm{~Hz}$ at $100 \mathrm{~Hz}$ excitation frequency. It must be mentioned, that this effect depends on the device electronics, and is not a limiting factor of ECT in general. The calculated deviation is $21.7 \%$. In general, for higher excitation frequency it is possible to increase the cutoff frequency, whereas the skin depth decreases, and thereby the ability to detect deep lying subsurface defects.

$$
N R M S D=\sqrt{\frac{\sum_{i=1}^{n}\left[y_{i, N u m}-y_{i, E x p}\right]^{2}}{\sum_{i=1}^{n}\left[y_{i, E x p}\right]^{2}}} 100 \%
$$

Figure 5 shows the measured and the simulated relative force perturbations of an artificial defect at a depth of $3 \mathrm{~mm}$ for velocities of $0.3,0.5$ and $0.7 \mathrm{~m} / \mathrm{s}$. The defect was detectable in all cases. The given velocities correspond to magnetic Reynolds numbers of $0.29,0.48$ and 0.67 and a linear dependency of the velocity can be assumed. Looking at the experimental results, velocity-proportional disturbances are observable in both force components. This results in a decreased detection performance and is a field of current research. However, there is a very good agreement between experimental and numerical results at low velocities with a NRMSE of $7.1 \%$ for the lift force, whereas the NRMSE increases up to $38.4 \%$ at $0.7 \mathrm{~m} / \mathrm{s}$ for the drag force.

\subsection{Defect depth study}

Figure 6 shows measured and simulated output signals obtained by ECT at different defect depths from $3 \mathrm{~mm}$ to $7 \mathrm{~mm}$, considering a testing velocity of $0.25 \mathrm{~m} / \mathrm{s}$ and ECT excitation frequency of $100 \mathrm{~Hz}$. This results in a skin-depth of $9.1 \mathrm{~mm}$, assuming a conductivity of the specimen of $30.61 \mathrm{MS} / \mathrm{m}$. The artificial defect was detectable up to a depth of $7 \mathrm{~mm}$ which indicates, that the spatial decay of the magnetic field is the limiting factor in this case. Again, a good agreement between measured and numerically calculated 


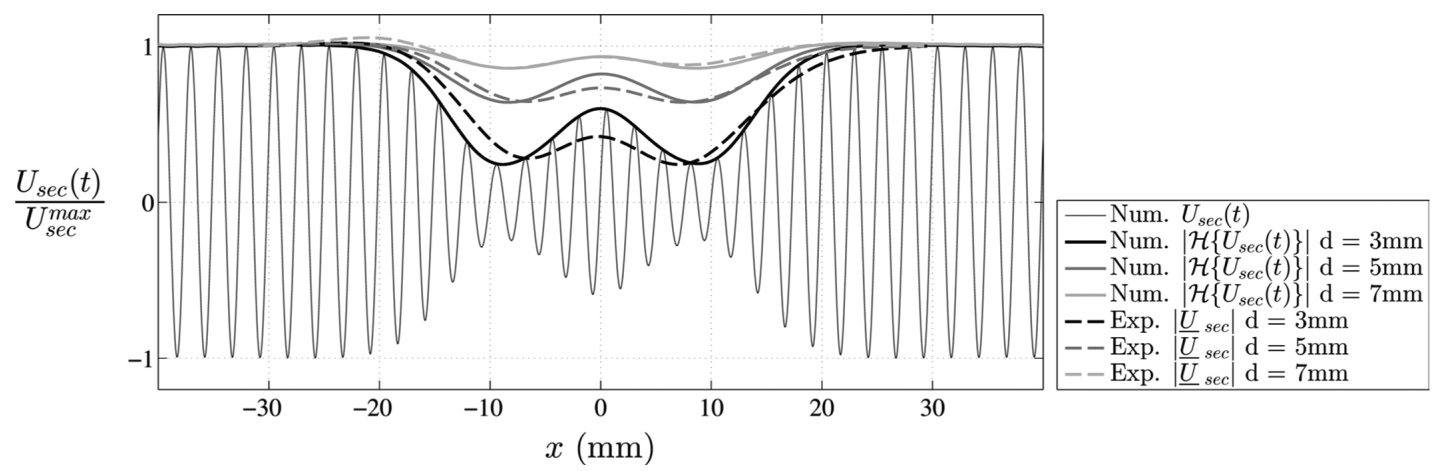

Fig. 6. Measurements and simulations of the normalized induced secondary voltage $U_{\text {sec }}(t)$ obtained with the ECT-probe PKA-48 for different defect depths in case of an artificial defect of size $12 \times 2 \times 2 \mathrm{~mm}^{3}$ at a testing velocity of $0.25 \mathrm{~m} / \mathrm{s}$.
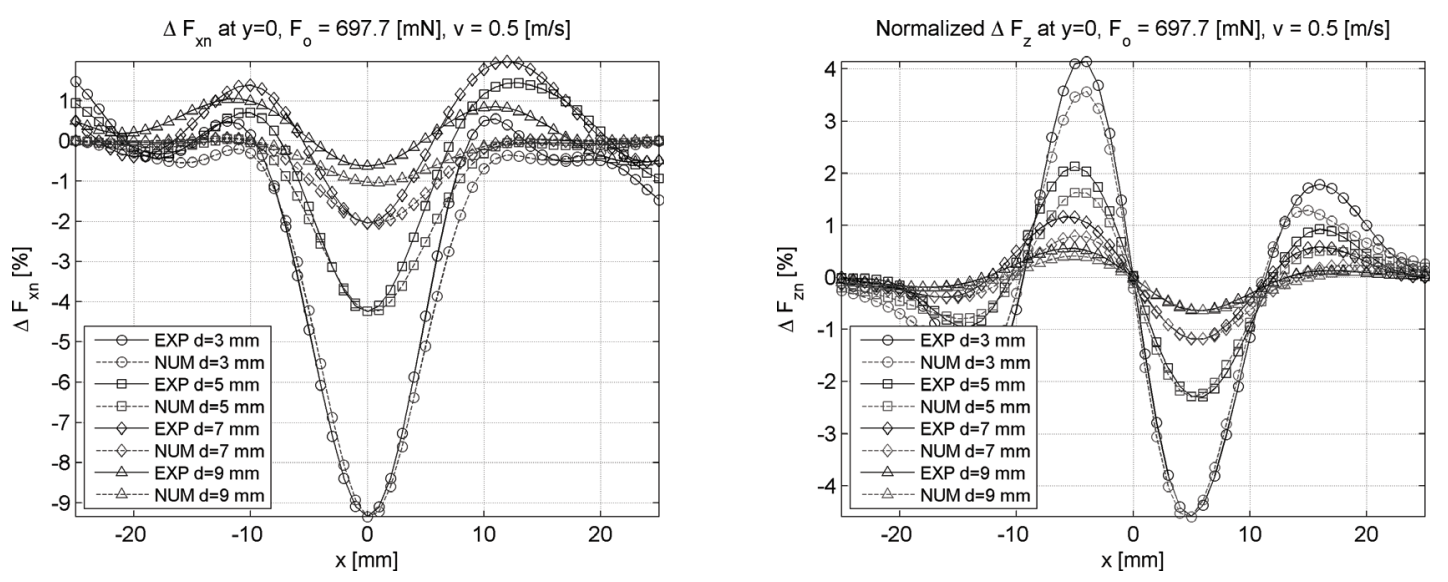

Fig. 7. Normalized forces exerting on the permanent magnet: Drag force $\Delta F_{x n}($ left $)$, lift force $\Delta F_{z n}($ right), measured in the vicinity of an artificial defect of size $12 \times 2 \times 2 \mathrm{~mm}^{3}$ for different depths at a testing velocity of $0.5 \mathrm{~m} / \mathrm{s}$.

signals can be observed, wherein the measured peak-to-peak ratio was always smaller or equal to the simulated signals.

A comparable study was performed for LET, where the testing velocity was $0.5 \mathrm{~m} / \mathrm{s}$ and the defect depth was varied between $3 \mathrm{~mm}$ and $9 \mathrm{~mm}$. The results are shown in Fig. 7. The artificial defect was detectable up to a depth of $9 \mathrm{~mm}$. The magnetic Reynolds number amounts to 0.48 which indicates no significant influence of motion evoked skin effect. The characteristics of LET to operate with magnetostatic fields is one advantage compared to ECT regarding the detection of subsurface defects.

However, for high magnetic Reynolds numbers, a distortion of the motion induced eddy current profile and asymmetric force profiles are observable. The calculated NRMSE for defect depth of $9 \mathrm{~mm}$ ranges from $3.5 \%$ for $\Delta F z n$ to $15.3 \%$ for $\Delta F x n$.

\section{Conclusion}

The present study compares the characteristics of traditional eddy current testing ECT to the new technique Lorentz force eddy current testing (LET). Investigations were performed in terms of velocity and defect depth. 
Numerical simulations revealed, that ECT can be applied for moving conductors as long as the analogue and digital signal processing unit is adjusted accordingly. It was found, that the Hilbert-transform is a suitable technique to resolve defects at higher testing speeds. Contra-intuitivly, no significant effects were observed regarding motion induced eddy currents in case of ECT, e.g. asymmetric output signals. Further investigations like in [4] are needed in the time-domain to investigate the interaction between motion and frequency induced eddy currents in high speed applications for different defects. In case of LET, relative motion is necessary to induce a sufficient amount of eddy currents. In the range of low magnetic Reynolds numbers $\left(R_{m}<1\right)$, a linear increase of force can be observed with increasing testing speeds, like in eddy current braking systems [6].

The frequency dependent penetration depth is the limiting factor of ECT to detect subsurface defects. Hence, the frequency was reduced to $100 \mathrm{~Hz}$, which creates the possibility to detect defects up to a depth of $7 \mathrm{~mm}$ at the described experimental setup. In case of LET, subsurface defects were detectable up to a depth of $9 \mathrm{~mm}$. The limiting factor of LET is given by a critical velocity or critical magnetic Reynolds number in the transition between the linear and nonlinear region, where the drag force experiences a maximum value. In this region motion induced eddy currents are pushed to the surface and a skin effect like behavior can be observed. Further studies will address this effect to quantify the possibilities of LET. In addition, a vibration analysis will be performed to reduce the observed oscillations in the force signals.

\section{Acknowledgment}

The present work is supported by the Deutsche Forschungsgemeinschaft (DFG) in the framework of the Research Training Group 1567 "Lorentz force velocimetry and Lorentz force eddy current testing".

\section{References}

[1] B. Auld and J. Moulder, Review of advances in quantitative eddy current nondestructive evaluation, Journal of Nondestructive Evaluation 18(1) (1999), 3-36.

[2] H. Brauer and M. Ziolkowski, Eddy current testing of metallic sheets with defects using force measurements, Serbian Journal of Electrical Engineering 5(1) (2008), 11-20.

[3] P.A. Davidson, An introduction to magnetohydrodynamics, Cambridge University Press, Cambridge, 2001, ISBN 0-52179487-04914.

[4] N. Ida, Modeling of velocity effects in eddy current applications, Journal of Applied Physics 63 (1988), 3007-3009.

[5] G. Mook, F. Michel and J. Simonin, Electromagnetic imaging using probe arrays, Strojniški vestnik - Journal of Mechanical Engineering 3 (2011).

[6] A. Singh, Theory of eddy-current brakes with thick rotating disk, Proceedings of the Institution of Electrical Engineers 124 (1977), 373-376

[7] R.P. Uhlig, M. Zec, H. Brauer and A. Thess, Lorentz force eddy current testing, a prototype model, Journal of Nondestructive Evaluation 31(4) (2012), 357-372.

[8] M. Zec, R.P. Uhlig, M. Ziolkowski and H. Brauer, Lorentz force eddy current testing: Modelling of permanent magnets in dynamic simulation using logical expressions, 8th Int, Conference on Computation in Electromagnetics, (CEM2011), Wrozlaw, 2011, 1-2.

[9] M. Zec, Theory and numerical modelling of lorentz force eddy current testing, Dissertation, Ilmenau University of Technology (2012). 